Conclusions New drugs which target a reduction in toxicities have the highest impact on patient prognosis, and such drugs are within reach of current candidate products.

\section{P3-S3.10 AN ASSESSMENT OF THE EFFECTS OF ANCILLARY SERVICES ON CD4 COUNTS AND VIRAL LOADS OF PEOPLE LIVING WITH HIV/AIDS}

doi:10.1136/sextrans-2011-050108.459

K Ball, R Parrish, A Thornton. University of Kentucky, Lexington, USA

Background The Ryan White Modernisation Care Act 2006 integrated medical case management with supportive social services to ensure that Persons Living with HIV/AIDS (PLWHA) from lower socioeconomic, marginalised groups, and those with mental health/ substance use issues are sustained in medical care. This study sought to establish the relationship between the CD4 and viral load of PLWHA and the receipt of ancillary services.

Methods A random sample of $\mathrm{N}=222$ subjects were drawn from the Bluegrass Care Clinic (BCC) database. The cohort was $67 \%$ white, $21.6 \%$ black, and $8.6 \%$ Hispanic with the majority of the subjects between 100 and 149\% of the poverty level. Self-reported HIV risk factors for the sample were $59.9 \%$ MSM, 28.4\% heterosexual, and $8.1 \%$ IDU. This sample adequately represents the population of the $\mathrm{BCC}$ as ethnicity, socioeconomic status and risk factors were similar for the clinic population as a whole. One way ANOVA tests were used to determine if the receipt of ancillary services by PLWHA in the BCC affects their viral loads and CD4 counts by using a multivariable analysis.

Results There was a statistically significant difference in mean CD4 counts when comparing between ethnicities. The ANOVA test showed a statistically significant relationship $(p=0.002)$ between Hispanic ethnicity and mean CD4 count, as subjects in the study that were of Hispanic origin had lower mean CD4 counts (305) than non-Hispanic whites (550) and non-Hispanic African Americans (500). There was also a statistically significant difference $(p=0.021)$ between the mean viral load of PLWHA having one to three mental health visits and PLWHA with no mental health visits at all.

Conclusion At the BCC, patients with adherence issues appear to be referred for mental health services, confirming the essential role of social workers on a multi-disciplinary HIV team. The BCC also has a Hispanic population with disproportionately lower CD4 counts. Implications for program improvement are to continue to target Hispanic patients with lower CD4 counts to retain them in medical care and improve adherence to ART medications. This study was limited by the fact that ancillary social services were lumped in with medical case management in the data set rather than delineated into individual service categories. Future research should use data sets that more accurately capture the various ancillary services provided to the sample subjects.

\section{P3-S3.11 ABSTRACT WITHDRAWN}

\section{P3-S3.12 TRANSMITTED HIV DRUG RESISTANCE MUTATIONS IN ONTARIO, CANADA, 2002-2009}

doi:10.1136/sextrans-2011-050108.461

${ }^{1} \mathrm{~A}$ Burchell, ${ }^{2} \mathrm{~A}$ Bayoumi, ${ }^{1} \mathrm{C}$ Major, ${ }^{1} \mathrm{~S}$ Gardner, ${ }^{3} \mathrm{D}$ Taylor, ${ }^{4} \mathrm{~A}$ Rachlis, ${ }^{5} \mathrm{P}$ Sandstrom, ${ }^{1} S$ Rourke, ${ }^{5} \mathrm{~J}$ Brooks. ${ }^{1}$ Ontario HIV Treatment Network, Toronto, Canada; ${ }^{2} S t$. Michael's Hospital, Toronto, Cape Verde; ${ }^{3}$ Canadian AIDS Treatment Information Exchange, Toronto, Canada; ${ }^{4}$ Sunnybrook Health Sciences Centre, Toronto, Canada; ${ }^{5}$ Public Health Agency of Canada, Ottawa, Canada

Background We estimated the prevalence of transmitted HIV drug resistance (TDR) among HIV-positive outpatients in Ontario,
Canada who were diagnosed since 2002 when the provincial laboratories started testing for drug resistance among treatment-naïve patients.

Methods We analysed data from the Ontario HIV Treatment Network Cohort Study, a multi-site open dynamic cohort of people living with HIV. Participants were recruited from specialised HIV clinics and primary care practices. Data were obtained from medical chart extractions, interviews and linked with data from the Ontario Public Health Laboratories, which performs almost all viral load and genotypic resistance testing (GRT). We analysed data from participants who received GRT testing while treatment-naïve, defined as (1) no record of antiretroviral use in their clinical chart and (2) detectable viral load. We used the Stanford University HIV Drug Resistance Database to identify TDR mutations in the viral sequences of the protease and reverse transcriptase genes. We used descriptive statistics to characterise the prevalence of TDR mutations and report results with 95\% CI.

Results Among 623 persons diagnosed in 2002-2009, 330 received GRT while treatment naïve. Among those tested, the mean age was 39 (SD 9.9); 12\% were female, 65\% men who have sex with men (MSM), and $66 \%$ white. The median baseline viral load count was $4.5 \log 10$ copies/ml (IOR 3.9-5.0) and the median baseline CD4 count was 399 cells $/ \mathrm{mm}^{3}$ (IOR 240-540). Overall, 13.6\% (CI 9.9 to $17.3 \%$ ) had one or more drug resistance mutations, and $8.8 \%$ (CI 5.7 to $11.8 \%$ ), $4.8 \%$ (CI 2.5 to $7.2 \%$ ) and $2.7 \%$ (CI 1.0 to $4.5 \%$ ) had mutations conferring resistance against nucleoside/tide reverse transcriptase inhibitors (NRTIs), non-nucleoside reverse transcriptase inhibitors (NNRTIs), or protease inhibitors (PIs), respectively. TDR against two or more drug classes was observed in $2.7 \%$ (CI 1.0 to $4.5 \%$ ). The most common mutations were T215 revertants, M41L, and $\mathrm{K} 103 \mathrm{~N}$ in the RT gene. The proportion with TDR was highest among IDU (30.4\%), intermediate among MSM and heterosexuals $(12.0 \%$ and $14.3 \%$, respectively) and lowest among persons from HIV endemic regions $(6.9 \%)(p=0.06)$. Participants diagnosed in 2008-2009 had a higher proportion of NRTI mutations $(18.2 \%$ vs $5.9 \%, p=0.0009)$ and NNRTI mutations $(11.7 \%$ vs $2.8 \%, p=0.004)$ than those diagnosed earlier; such increases were observed among MSM, heterosexuals and IDU. There was no evidence of a change in PI mutation frequency over time.

Conclusion Our finding of a recent increase in NRTI and NNRTI mutations is concerning but requires confirmation, ideally in a random sample of specimens from newly diagnosed individuals. Although the actual dates of infection were unknown, the results suggest that drug-resistant strains are commonly circulating within the established HIV epidemics in Ontario.

\section{Clinical sciences poster session 4: Human papillomavirus}

\section{P3-S4.01 HIGH-RISK HUMAN PAPILLOMAVIRUS (HR-HPV) INFECTION DETECTION IN RUSSIA: NEED TO INTENSIFY ITS LABORATORY PROFICIENCY WITH STANDARDISATION PROGRAMS?}

doi:10.1136/sextrans-2011-050108.462

${ }^{1} \mathrm{~V}$ Smelov, ${ }^{1} \mathrm{~A}$ Novikov, ${ }^{2} \mathrm{~S}$ Ouburg, ${ }^{2} \mathrm{~J}$ Pleijster, ${ }^{1} \mathrm{~A}$ Allambergenov, ${ }^{3} \mathrm{~V}$ Revzon, ${ }^{3}$ A Gorelov, ${ }^{2}$ S Morre. ${ }^{1}$ Medical Academy of Postgraduate Studies, St. Petersburg, Russian Federation; ${ }^{2}$ VU University Medical Center, Amsterdam, Netherlands; ${ }^{3}$ St Petersburg State University, St. Petersburg, Russian Federation

Introduction HR-HPV infection is one of the most often diagnosed viral STIs and has been linked to some anogenital malignances. HPV vaccines have not yet widely been approved for men, representing an important source of HPV transmission between sex partners. The earliest detection plays a crucial role in decreasing of a number of HR-HPV-infected (wo)men and some screening 